\title{
Pleuro-Pulmonary Complications of Rheumatoid Arthritis
}

\author{
John P Corcoran MRCP, Mehreen Ahmad MBBS, Rahul Mukherjee FRCP, and \\ Karen C Redmond MD FRCS (CTh)
}

\section{Introduction}

Rheumatoid arthritis (RA) is a chronic systemic inflammatory disease whose distinctive feature is the development of symmetrical polyarthritis. It has a prevalence of $\sim 1 \%$ in the general population, predominating in females and peaking between the ages of 30 and 50 years; it is found worldwide, although some ethnic groups show an increased propensity for the disease. The burden of illness in these patients is manifest in a mortality rate that is more than twice that of age-matched individuals in the general population, with poor prognostic factors including rheumatoid factor seropositivity, anemia of chronic disease, presence of anti-cyclic citrullinated peptide antibodies, and extra-articular manifestations. Ellman and Ball ${ }^{1}$ first described lung disease in association with RA, and since that time, various pulmonary manifestations, including pleural effusions, lung nodules, obstructive lung disease, and vasculitis, have been described in the literature. In addition to problems caused by the disease, immunosuppressant agents and other medications used to treat RA can be directly pneumotoxic, predispose the patient to opportunistic infection, and impair wound repair or healing. Clinical features associated with RA-associated lung disease include male gender, rheumatoid factor seropositivity, severe articular disease, subcutaneous nodules, and other extra-articular manifestations.

Dr Corcoran is affiliated with the Oxford Centre for Respiratory Medicine, Churchill Hospital, Oxford, UK. Drs Corcoran, Ahmad, and Mukherjee are affiliated with the Department of Respiratory Medicine, Milton Keynes Hospital, Milton Keynes, UK. Dr Mukherjee is affiliated with the Department of Respiratory Medicine and Physiology, Birmingham Heartlands Hospital, Birmingham, UK. Dr Redmond is affiliated with the Department of Thoracic Surgery, Mater Misericordiae University Hospital, Dublin, Ireland.

The authors have disclosed no conflicts of interest.

Correspondence: Dr John P Corcoran, Oxford Centre for Respiratory Medicine, Churchill Hospital, Oxford OX3 7LE, United Kingdom. Email: john.corcoran@ouh.nhs.uk.

DOI: $10.4187 /$ respcare.02597
Progressive nodular and pleural lung disease associated with RA represents a particular clinical challenge. The nodules are often multiple and involve both lungs, varying in size from a few millimeters to several centimeters. A propensity for the lung peripheries and fissures with an ability to become necrotic and rupture results in complications such as recurrent secondary pneumothorax, bronchopleural fistula formation with sizeable air leak, hemorrhage, pulmonary abscess formation, and empyema. The inflammation, fibrous visceral peel formation (with trapped lung) and noncompliant parenchyma associated with rheumatoid lung mean achieving key clinical objectives such as closure of the air leak, obliteration of the pleural space, eradication of infection, and effective pleurodesis may prove impossible. An additional tendency for the pleuropulmonary complications of RA to recur can result in disease that requires repeated intervention and is resistant to standard therapy. This case series illustrates the challenges that may occur in severe pleuro-pulmonary disease associated with RA, alongside a review of the literature relating to this area of practice.

\section{Case Summaries}

\section{Case 1}

A 51-year-old woman presented to A\&E in June 2008 with acute confusion. Her medical history included seropositive RA diagnosed 10 years previously and managed with leflunomide $20 \mathrm{mg}$ daily and prednisolone $2.5 \mathrm{mg}$ daily. A plain chest radiograph demonstrated opacification of the right hemithorax with a computed tomography (CT) scan confirming a large right pleural effusion, mediastinal lymphadenopathy, and multiple pulmonary nodules that were presumed to be secondary to rheumatoid lung disease. The effusion was drained under ultrasound guidance, and the patient was treated for microbiologically confirmed empyema with antibiotics. A follow-up CT scan in July showed a small right pneumothorax that required drainage 1 week later in the context of worsening dyspnea and increasing size. An interval CT scan after 2 weeks demonstrated minimal residual pneumothorax, and, as the pa- 
tient was asymptomatic, further intervention was deemed inappropriate.

In September 2008 the patient presented again with dyspnea on minimal exertion. A CT scan demonstrated a large right hydropneumothorax associated with a ruptured pulmonary nodule, and following drain insertion for symptom relief the patient was referred for surgical intervention. A right video-assisted thoracoscopic surgery (VATS) exploration with talc pleurodesis was performed; fluid culture was negative, and biopsy samples confirmed the presence of rheumatoid pleuritis and nodular disease. Recovery was uneventful, and the patient went home after 8 days with a wide-bore drain and Heimlich valve in situ to manage the ongoing air leak.

At out-patient follow-up over the next 3 months persistent air leak from a bronchopleural fistula meant the drain was left in position. This was eventually complicated by chronic empyema and breakdown of the subscapular VATS port site, requiring a prolonged course of oral ciprofloxacin. In the context of persistent pyopneumothorax and air leak an unsuccessful attempt was made in February 2009 to resolve the problem with a chest drain change, chemical pleurodesis (doxycycline and povidone-iodine), and application of negative pressure to the drain. The patient was left with an ongoing air leak and 20\% residual pneumothorax with wide-bore drain and Heimlich valve in situ.

Over the next year the patient had her right-sided chest drain changed three times (twice for dislodgement, once for blockage), as well as experiencing a left-sided pneumothorax secondary to another ruptured rheumatoid nodule that resolved with simple drainage. In an attempt to reduce the risk of infection her prednisolone and leflunomide treatment were stopped. Despite this, the patient required recurrent treatment for persistent pleural space infection. On the last of these occasions she required ICU admission for septic shock where, in the context of multiorgan failure including difficult ventilation due to bilateral bronchopleural fistulae, the patient died 2 years after her first presentation.

\section{Case 2}

A 64-year-old man was referred to the respiratory clinic in May 2008 with progressive dyspnea. He had received a diagnosis of RA in 1976, and his condition was managed with methotrexate $7.5 \mathrm{mg}$ weekly, leflunomide $20 \mathrm{mg}$ daily, and prednisolone $5 \mathrm{mg}$ daily. Other medical problems included significant cardiac disease with atrial fibrillation, bioprosthetic aortic valve replacement, and dual-chamber pacemaker in situ; and a diagnosis of right basal pleural thickening of uncertain etiology made in 1998. A CT scan demonstrated right lower lobe bronchiectasis with thickwalled segmental cavitation indicative of recurrent or persistent infection, alongside widespread pleural thickening

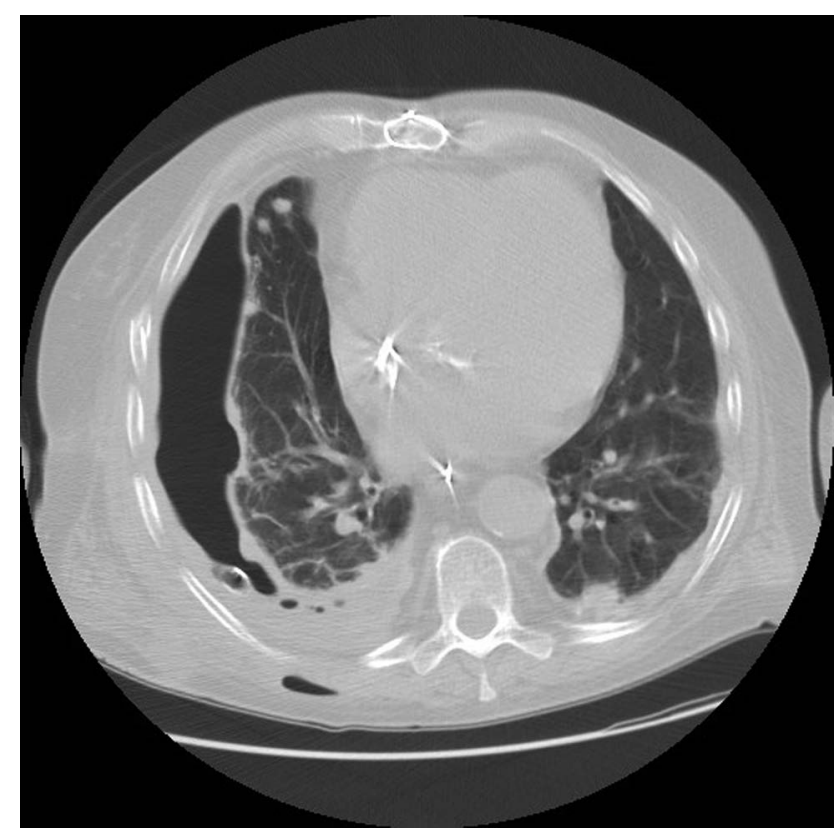

Fig. 1. CT image of Case 2 demonstrating right hydropneumothorax with multiple pulmonary nodules secondary to rheumatoid disease visible bilaterally.

with punctuated calcification suggesting chronic pleuritis. As there were no symptoms or biochemical markers of infection the patient's condition was managed conservatively.

He was subsequently admitted to the hospital in October 2008 with a week-long history of cough, dyspnea, and fever. A plain chest radiograph showed a right-sided hydropneumothorax that drained purulent fluid on drain insertion. After 1 week of treatment with intravenous antibiotics the chest drain continued to bubble, and after a CT scan (Fig. 1) confirmed the presence of persisting hydropneumothorax the patient was referred for surgery. Rigid bronchoscopy, right-sided thoracotomy, and decortication were performed; the postoperative period was difficult with persistent air leak and a requirement for vasopressor treatment. Intra-operative samples grew Aspergillus, and intravenous antifungal agents were added to ongoing antibiotic therapy. Three weeks after surgery the patient was discharged from the hospital receiving treatment with oral antibiotics and antifungal agents; however, a persistent right pneumothorax and contiguous lower lobe cavity required a wide-bore drain to be left in position. The patient was readmitted to the hospital 1 week later with rigors and restarted on therapy with intravenous antimicrobial agents, alongside weaning off methotrexate and steroids after discussion with his rheumatologist. Further surgical review concluded that rheumatoid pleurisy alongside significant air leak from the right lower lobe cavity and bronchopleural fistula would prevent successful pleurodesis and reexpansion of the lung. The patient was allowed to go home 
with a chest drain in situ once his symptoms and inflammatory markers settled.

At subsequent follow-up over the next year the patient remained stable from a respiratory perspective. Repeat imaging, including cross-sectional studies, demonstrated an unchanged trapped right lung with thick-walled right lower lobe cavitation, bronchopleural fistula, and persistent pneumothorax. His condition continued to be managed with a shortened wide-bore chest drain attached to a colostomy bag with de-airing holes; management options, including thoracic window formation or muscle flap to fill the defect, were discussed and either declined or deemed inappropriate due to concerns regarding the patient's fitness for general anesthetic. The patient died from sequelae of his cardio-respiratory disease 2 years after his initial referral.

\section{Case 3}

A 55-year-old woman was referred to the respiratory clinic in September 2005 after a plain chest radiograph demonstrated an incidental left-sided hydropneumothorax. She had seropositive RA diagnosed in 1987 requiring multiple joint replacements and cervical spine surgery; alongside other autoimmune conditions including Sjögren syndrome, vitiligo, and hypothyroidism. She was receiving treatment with methotrexate $15 \mathrm{mg}$ weekly and anti-tumor necrosis factor alpha (TNF- $\alpha$ ) therapy (adalimumab) with concurrent isoniazid prophylaxis. Cross-sectional imaging in 2003 had demonstrated lung nodules that were presumed secondary to rheumatoid disease, while the patient reported drainage of a left-sided pleural effusion in 2004. Ultrasound-guided aspiration of the left hydropneumothorax obtained cloudy fluid with $\mathrm{pH}>7.2$ and glucose $>4$ $\mathrm{mmol} / \mathrm{L}$, while the results of a bacterial culture were negative. In the absence of systemic symptoms conservative management was pursued. Routine follow-up over the next 2 years was uneventful with stable appearance of the left hydropneumothorax on serial imaging.

In January 2008 the patient presented acutely with malaise and dyspnea. A plain chest radiograph demonstrated an enlarged left hydropneumothorax that subsequently drained purulent fluid. Treatment with adalimumab and methotrexate were stopped, and treatment with broadspectrum antibiotics commenced for 6 weeks. The patient required readmission to the hospital in late March with dyspnea and discharge from the previous drain site. A pleural fluid sample obtained in January had cultured $M y$ cobacterium kansasii, and in discussion with a microbiologist the patient started treatment with oral rifampicin, ethambutol, and clarithromycin. An ultrasound-guided chest drain was inserted for treatment of the residual left pleural effusion, and a surgical opinion was sought; the surgeon felt that early intervention might prove beneficial given the presence of a persistent hydropneumothorax,

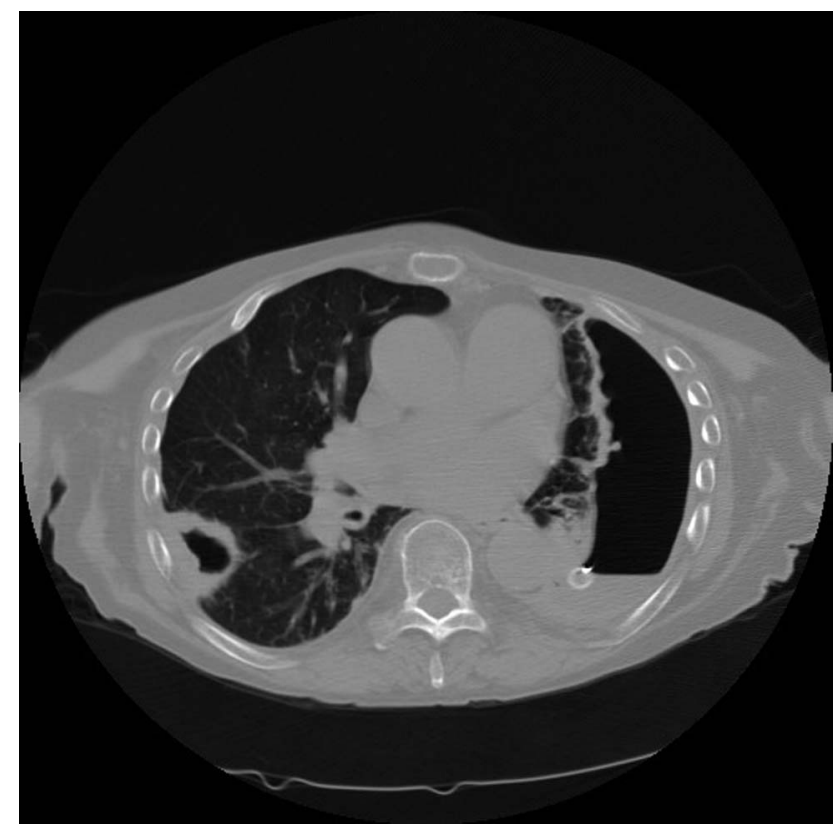

Fig. 2. CT image of Case 3 with left hydropneumothorax and drain in situ, near a large cavitating right rheumatoid pulmonary nodule.

empyema, and bronchopleural fistula identified on a CT scan (Fig. 2).

In early April the patient underwent flexible (fiberoptic) bronchoscopy, left rib resection, and wide-bore chest drain insertion before being transferred back to her local respiratory unit for ongoing in-patient care. Unfortunately the chest drain became dislodged after 2 weeks, and, in the context of accumulating pleural fluid and rising levels of inflammatory markers, the patient was referred for further surgical input. A VATS exploration, drainage, and washout of the pleural cavity were performed with the drain left in situ on hospital discharge and a plan to complete 12 months of anti-mycobacterial treatment.

Over the next 18 months the patient required two further hospital admissions to treat complications of chronic pleural infection despite continuing not to receive immunosuppression therapy. Serial imaging demonstrated gradual improvement in the persistent left hydropneumothorax with the drain in situ, although plans for thoracic window formation were prevented by the patient's limited functional reserve. A steady physical decline culminated in hospital admission in late 2010 when, in the context of type 2 respiratory failure, sepsis, and cardiac ischemia, the patient died.

\section{Discussion}

This case series demonstrates the difficulties that can occur with severe pleuro-pulmonary disease associated with RA and escalating management approaches, as well as the 
morbidity and mortality associated with this process. It is difficult to be certain about the proportion of patients with RA in whom pulmonary manifestations develop as many patients may be asymptomatic or never undergo relevant investigations. While RA is three times more common in females, it is males in whom pulmonary complications proportionally develop more often, a key observation because recent data suggest the incidence of RA-associated lung disease is on the rise, ${ }^{2}$ and represents a major cause of morbidity and mortality in these patients..$^{3,4}$

Postmortem series suggest that RA-associated pleural disease may occur in between 38 and $73 \%$ of patients over a lifetime, ,3,5,6 although observational work has estimated the annual incidence of pleural effusion formation in patients with RA at $0.34 \%$ for women and $1.54 \%$ for men. ${ }^{7}$ The development of pleural disease can occur alongside or precede the first signs of joint involvement ${ }^{8,9}$ and like other connective tissue diseases may have a genetic predisposition. ${ }^{10}$ Despite being the most common intra-thoracic manifestation of RA, symptoms of pleural disease will never develop in the majority of patients; only a reported $20 \%$ of individuals with RA experience pleurisy at any point in time, ${ }^{9}$ whereas overt clinical evidence of pleural disease is found in $<5 \%$ of patients with RA. ${ }^{11,12}$

The presence of a pleural effusion in a patient with RA should prompt diagnostic investigation, with ultrasoundguided thoracocentesis being the first-line test. Pleural fluid in RA is usually exudative, non-odorous, and often turbid with a low $\mathrm{pH}(<7.3)$ and glucose level $(<50 \mathrm{mg} / \mathrm{dL}$ or $2.8 \mathrm{mmol} / \mathrm{L}$ ), whereas more longstanding effusions may become chyliform. ${ }^{13,14}$ The presence of rheumatoid factor in pleural fluid reflects serum levels and is highly suggestive of an RA-associated etiology, whereas the cytological appearance may be characteristic. ${ }^{7,15,16}$ If the clinical history and thoracocentesis results fail to confirm a diagnosis, medical thoracoscopy or VATS and pleural biopsy may be necessary, particularly when there is concern regarding occult malignancy or infection. In cases of rheumatoid pleuritis both the "gritty" frozen macroscopic appearance of the pleura and histopathology are classically diagnostic. ${ }^{17,18}$ Most rheumatoid effusions are ultimately self-limiting with over twothirds resolving within 4 months of their diagnosis. ${ }^{9}$

RA-associated pleural effusion shares many biochemical and macroscopic features with empyema, and the clinical history should be considered alongside appropriate microbiological studies before starting treatment for infection. The risk of developing empyema in association with RA is unclear ${ }^{9,17,19}$ but is certainly higher with immunosuppression therapy and treatment with disease-modifying anti-rheumatic drugs; patients receiving anti-TNF- $\alpha$ therapy are at risk for mycobacterial infection and should be specifically screened for this. The presence of other RAassociated pulmonary disease, such as bronchiectasis or rheumatoid lung nodules, may predispose the patient to infection by providing a portal of entry to the pleural space-for instance through the rupture and formation of a bronchopleural fistula. ${ }^{20}$ It may be necessary to review any prescribed immunosuppression therapy; whereas conventional wisdom dictates that treating RA reduces the risk of progressive pulmonary disease, excessive immunosuppression therapy may make treating an infection impossible. Furthermore, continuing treatment with any drug having an anti-inflammatory effect may negatively impact on the likelihood of successful pleurodesis following treatment for empyema or pneumothorax, ${ }^{21,22}$ with talc preferable to complete pleurectomy, decortications, or other chemical agents. There is increasing evidence linking specific drugs, notably leflunomide, with accelerated pulmonary nodulosis, ${ }^{23-26}$ which may complicate management further.

Surgical intervention may be necessary in patients with rheumatoid lung disease for reasons including the investigation of lung nodules or pleural effusion, and the treatment of pneumothorax, empyema, or symptomatic pleural thickening. There is no consensus guidance on how to approach these cases, and consequently clinical decision making is based on the experience of the individual responsible clinician. The difficulty in managing these cases has long been recognized, with the formation of fibrous peel in association with chronic rheumatoid pleuritis, making it difficult to divide away the lung during decortication and close any sites of air leak. ${ }^{27}$ Although the surgical treatment of primary and secondary spontaneous pneumothorax using thoracotomy or VATS is highly efficacious in the general population, ${ }^{28,29}$ this may not apply in patients with RA-associated pleuro-pulmonary disease in whom the underlying lung can be grossly abnormal. Although guidelines for the management of both pneumothorax and empyema recommend small-bore chest tube drainage as part of standard first-line therapy, ${ }^{30,31}$ it has been suggested that a more aggressive strategy using early surgical intervention with a concerted effort to re-expand the lung, obliterate the pleural space, and facilitate pleurodesis might benefit patients already identified as having abnormal pleura secondary to RA. ${ }^{32}$

This case series describes the management and longerterm outcome in a complex group of patients with RA, in whom, as a consequence of their underlying disease and the medications used to treat it, the development of a range of pleuro-pulmonary complications occurred that were resistant to conventional therapy. Whatever therapeutic approach is taken the patient must be counseled on the increased risk of complications and failure of any intervention; the clinical team should also be prepared with alternative options that might prove necessary-these may include long-term chest drain insertion or thoracic window formation with or without delayed closure for control of persistent air leak or empyema-to maximize potential success in managing the patient. 


\section{Pleuro-Pulmonary Complications of Rheumatoid Arthritis}

\section{Teaching Points}

- Pleural involvement with RA is common, with most patients being likely to remain asymptomatic or recover spontaneously. Significant manifestations include nodule formation and rupture, causing bronchopleural fistulae and/or secondary pneumothorax; lung abscess; and empyema.

- Complex RA-associated pleuro-pulmonary disease can be difficult to treat, given its tendency to recur, the physical characteristics of the underlying lung, and an immunosuppressed state that predisposes the patient to chronic infection and poor healing.

- Complex and symptomatic RA-associated pleuro-pulmonary disease is associated with significant morbidity and mortality. Patients should be counseled on the increased risk of complications and failure for any therapeutic intervention, whereas clinicians should consider what long-term options are available in case initial management is unsuccessful.

\section{ACKNOWLEDGMENTS}

The authors thank Dr Milan Bhattacharya (Milton Keynes Hospital, United Kingdom) and Dr Najib Rahman (Churchill Hospital, Oxford, United Kingdom) for their assistance in the preparation of this article.

\section{REFERENCES}

1. Ellman P, Ball RE. Rheumatoid disease with joint and pulmonary manifestations. BMJ 1948;2(4583):816-820.

2. Bartels CM, Bell CL, Shinki K, Rosenthal A, Bridges AJ. Changing trends in serious extra-articular manifestations of rheumatoid arthritis among United States veterans over 20 years. Rheumatology (Oxford) 2010;49(9):1670-1675.

3. Toyoshima H, Kusaba T, Yamaguchi M. Cause of death in autopsied RA patients. Ryumachi 1993;33(3):209-214.

4. Sihvonen S, Korpela M, Laipalla P, Mustonen J, Pasternack A. Death rates and causes of death in patients with rheumatoid arthritis: a population-based study. Scand J Rheumatol 2004;33(4):221-227.

5. Rosenberg EF, Baggenstoss AH, Hench PS. The causes of death in thirty cases of rheumatoid arthritis. Ann Intern Med 1944;20(6):903919.

6. Talbott JA, Calkins E. Pulmonary involvement in rheumatoid arthritis. JAMA 1964;189(12):911-913.

7. Jurik AG, Graudal H. Pleurisy in rheumatoid arthritis. Scand J Rheumatol 1983;12(2):75-80.

8. Horler AR, Thompson M. The pleural and pulmonary complications of rheumatoid arthritis. Ann Intern Med 1959;51(6):1179-1203.

9. Walker WC, Wright V. Rheumatoid pleuritis. Ann Rheum Dis 1967; 26(6):467-474.

10. Hakala M, Tiilikainen A, Hämeenkorpi R, Ilonen J, Jalava S, Ruuska P, Mäkitalo R. Rheumatoid arthritis with pleural effusion includes a subgroup with autoimmune features and HLA-B8, Dw3 association. Scand J Rheumatol 1986;15(3):290-296.

11. Hyland RH, Gordon DA, Broder I, Davies GM, Russell ML, Hutcheon MA, et al. A systematic controlled study of pulmonary abnormalities in rheumatoid arthritis. J Rheumatol 1983;10(3):395-405.

12. Sahn SA. Pathogenesis of pleural effusions and pleural lesions. In: Cannon GW, Zimmerman GA, editors. The lung in rheumatic dis- eases. Lung biology in health and diseases, Vol 45. New York: Marcel Dekker;1990:27-48.

13. Lillington GA, Carr DT, Mayne JG. Rheumatoid pleurisy with effusion. Arch Intern Med 1971;128(5):764-768.

14. Hunder GG, McDuffie FC, Hepper NG. Pleural fluid complement in systemic lupus erythematosus and rheumatoid arthritis. Ann Intern Med 1972;76(3):357-363.

15. Halla JT, Schrohenloher RE, Volanakis JE. Immune complexes and other laboratory features of pleural effusions: a comparison of rheumatoid arthritis, systemic lupus erythematosus, and other diseases. Ann Intern Med 1980;92(6):748-752.

16. Naylor B. The pathognomonic cytologic picture of rheumatoid pleuritis: the 1989 Maurice Goldblatt Cytology award lecture. Acta Cytol 1990;34(4):465-473.

17. Jones FL Jr., Blodgett RC Jr. Empyema in rheumatoid pleuropulmonary disease. Ann Intern Med 1971;74(5):665-671.

18. Faurschou P, Francis D, Faarup P. Thoracoscopic, histological, and clinical findings in nine cases of rheumatoid pleural effusion. Thorax 1985;40(5):371-375

19. Dieppe PA. Empyema in rheumatoid arthritis. Ann Rheum Dis 1975; 34(2):181-185

20. Hindle W, Yates DA. Pyopneumothorax complicating rheumatoid lung disease. Ann Rheum Dis 1965;24(1):57-60.

21. Xie C, Teixeira LR, McGovern JP, Light RW. Systemic corticosteroids decrease the effectiveness of talc pleurodesis. Am J Respir Crit Care Med 1998;157(5):1441-1444.

22. Hunt I, Teh E, Southon R, Treasure T. Using non-steroidal antiinflammatory drugs (NSAIDs) following pleurodesis. Interact Cardiovasc Thorac Surg 2007;6(1):102-104.

23. Cunnane G, Warnock M, Fye KH, Daikh DI. Accelerated nodulosis and vasculitis following etanercept therapy for rheumatoid arthritis. Arthritis Rheum 2002;47(4):445-449.

24. Rozin A, Yigla M, Guralnik L, Keidar Z, Vlodavsky E, Rozenbaum M, et al. Rheumatoid lung nodulosis and osteopathy associated with leflunomide therapy. Clin Rheumatol 2006;25(3):384-388.

25. van Ede A, den Broeder A, Wagenaar M, van Riel P, Creemers MC. Etanercept-related extensive pulmonary nodulosis in a patient with rheumatoid arthritis. J Rheumatol 2007;34(7):1590-1592.

26. Kim SH, Yoo WH. Recurrent pneumothorax associated with pulmonary nodules after leflunomide therapy in rheumatoid arthritis: a case report and review of the literature. Rheumatol Int 2011;31(7):919-922.

27. Yarbrough JW, Sealy WC, Miller JA. Thoracic surgical problems associated with rheumatoid arthritis. J Thorac Cardiovasc Surg 1975; 69(3):347-354

28. Chambers A, Scarci M. In patients with first-episode primary spontaneous pneumothorax is video-assisted thoracoscopic surgery superior to tube thoracostomy alone in terms of time to resolution of pneumothorax and incidence of recurrence? Interact Cardiovasc Thorac Surg 2009;9(6):1003-1008.

29. Shaikhrezai K, Thompson AI, Parkin C, Stamenkovic S, Walker WS. Video-assisted thoracoscopic surgery management of spontaneous pneumothorax-long-term results. Eur J Cardiothorac Surg 2011; 40(1):120-123

30. Davies HE, Davies RJ, Davies CW; BTS Pleural Disease Guideline Group. Management of pleural infection in adults: British Thoracic Society Pleural Disease Guideline 2010. Thorax 2010;65(Suppl 2): ii41-ii53.

31. MacDuff A, Arnold A, Harvey J; BTS Pleural Disease Guideline Group. Management of spontaneous pneumothorax: British Thoracic Society Pleural Disease Guideline 2010. Thorax 2010;65(Suppl 2): ii18-ii31.

32. Rueth N, Andrade R, Groth S, D'Cunha J, Maddaus M. Pleuropulmonary complications of rheumatoid arthritis: a thoracic surgeon's challenge. Ann Thorac Surg 2009;88(3):e20-e21. 